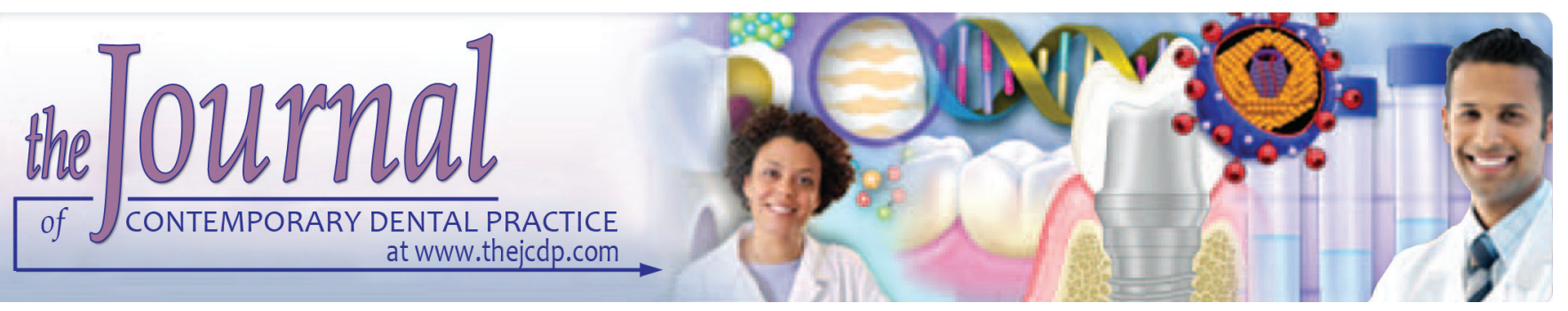

\title{
Treatment of Osteoradionecrosis of the Jaw with Ozone in the Form of Oil-based Gel: 1-year follow-up
}

\author{
${ }^{1}$ Edoardo Bianco, ${ }^{2}$ Marcello Maddalone, ${ }^{3}$ Gianluca Porcaro, ${ }^{4}$ Ernesto Amosso, ${ }^{5}$ Marco Baldoni
}

\begin{abstract}
Aim: Osteoradionecrosis of the jaws (ORNJs) is aseptic bone necrosis that develops in post-irradiated bone tissue of patients who underwent radiotherapy for head-neck tumors. The present study aims to clinically assess the regenerative ability of the ozone in the form of oil-based gel applied to the exposed bone area in the treatment of ORNJ.
\end{abstract}

Materials and methods: Eight patients who underwent radiotherapy for the treatment of cervical or neck cancer were diagnosed with ORN of the jaws at our Department, for a total of 11 sites of necrotic bone exposure ( 3 patients were diagnosed with more than one site of osteoradionecrosis). In the therapeutic protocol, the exposed bone lesion and osteomucosal margin were cleaned with manual debridement. Then the ORN lesion was treated with topical applications of ozone delivered as oil suspension (Ozosan $®$ - Sanipan, Clivio, Italy) on the exposed bone for 10 minutes. The application was repeated each week until movement of the necrotic fragment was noted

Results: In six patients on 8 (75\%) lesions resolved with complete mucosal healing with 3 to 19 ozone applications. Total sequestration of the necrotic bone with spontaneous expulsion was observed. One patient improved his conditions shifting from a stage B2S1 to B1S1 according to He et al. classification. A patient only worsened his conditions with treatment. No toxicity was reported or observed.

Conclusion: These results showed the efficacy of ozone oil suspensions in the non-invasive treatment of ORN, probably related to its properties of stimulation of local revascularization and antibacterial activity, and the good tolerability of the related protocol used.

1,3-5 Department of Surgery and Translational Medicine, University of Milano Bicocca, Monza, Italy

${ }^{2}$ Department of Orthodontics, University of Milano Bicocca, Monza, Italy

Corresponding Author: Edoardo Bianco, Department of Surgery and Translational Medicine, University of Milano Bicocca, Monza, Italy, Phone: 03487268209, e-mail: edoardo. bianco13@gmail.com
Clinical significance: The use of this kind of medication should be included in ORNJ treatments as effective, noninvasive and self-administered.

Keywords: Osteoradionecrosis, Ozone, Radiotherapy, Medical oncology, Radiation.

How to cite this article: Bianco E, Maddalone M, Porcaro G, Amosso E, Baldoni M. Treatment of Osteoradionecrosis of the Jaw with Ozone in the Form of Oil-based Gel: 1-year follow-up. J Contemp Dent Pract 2019;20(2):270-276.

Source of support: Nil

Conflict of interest: None

\section{INTRODUCTION}

Radiotherapy is usually used as adjuvant therapy following oncological surgery, and it can be administered together with chemotherapy or as palliative treatment. ${ }^{1}$

Osteoradionecrosis (ORN) of the maxilla is one of the most severe and important complications of radiotherapy administered for head and neck tumors. It is characterized by an area of exposed necrotic bone in one or more locations of the upper or lower jaw, with failure to heal during at least three months in the absence of local neoplastic disease. ${ }^{1-4}$ This kind of complication could also happen when the immune system of patients is compromised like in hematologic disorders when dental devices are used. ${ }^{5-9}$ This kind of risk is also the reason why some radiographic diagnostic high-resolution machines could not be applied to living patient. ${ }^{10-12}$

It develops due to progressive hypoxia, hypovascularization, and hypo-cellularity of the affected bone. Radiation causes inflammation of small blood vessels (endoarteritis) resulting in the development of thrombi and occlusion of the vascular lumen. It also produces an increase in free radicals and causes altered collagen synthesis. 13,14 Compromised tissue perfusion and impaired repair and remodeling capacity make the bone vulnerable to necrosis. A minimal external trauma at this 
point can cause ulceration, facilitating contamination and infection, and thus favoring bone necrosis. ${ }^{15}$

The exposed part of the bone shows visible changes in color and strength, and radiologically a typical loss of bone structure developing into diffuse radio transparency corresponding to bone breakdown. ${ }^{16}$

Regaud first described it in 1922, and it is most commonly located in the mandible, probably due to the relatively poor vascularization of the lower jaw. ${ }^{17}$ When located in the upper jaw, the ORN develops less progressively, and the defects are less severe. ${ }^{18,19}$ It can develop spontaneously in one-third of all cases correlated with high doses of radiations (> 65 Gy), but usually appear after tooth extraction or oral surgery, due periodontal and apical disease or as a consequence to direct trauma caused by poorly fitting dentures. ${ }^{1,20,21}$

Extraction or oral invasive treatment before or after irradiation is said to be the most common initiating factor in the development of ORN in irradiated jaws, and the incidence of ORN after tooth extraction in irradiated patients is estimated to be around $2-18 \% .^{22,23}$

The latest classification for ORN introduced in 2015 by $\mathrm{HE}$ et al. differentiate bone and soft tissue lesions and classify them in multiple stages. ${ }^{24}$ Development of antibiotic resistance and the need to find new antimicrobial agents that would promote the activity of angiogenesis generated research into the use of ozone in the form of oil-based gel as a topical treatment of skin or mucous wounds.

Ozone can be used in three forms: oxygen/ozone gas, ozonated oil/gel, ozonated water. Ozonated oil has the ability to entrap ozone and then release it when in contact with a lesion. Ozonated oil/gel must be kept at low temperature $\left( \pm 2^{\circ} \mathrm{C}\right)$ so that $\mathrm{O}_{3}$ fills all of the polyunsaturated fatty acids in the oils. ${ }^{3}$ At room temperature, $\mathrm{O}_{3}$ tends to separate from the oil, while freezing polyunsaturated fatty acids leads to an increase in their tensile strengths, forming a molecular mesh that entraps ozone and prevents it from migration. Ozone is useful in medicine due to its antimicrobial activity against bacteria, viruses, protozoa, and fungi. Ozone also can stimulate blood circulation and the immune response. Various applications for ozone have been proposed in dentistry, including the elimination of bacterial pathogens, periodontal pocket disinfection and osseous disinfection, prevention of dental caries and tooth sensitivity, endodontic treatment, pain control, infection control, accelerated healing, tissue regeneration, temporomandibular joint treatment and control of halitosis. ${ }^{25}$ A more recent application of ozone is in the treatment of bisphosphonate-related osteonecrosis of the jaw (BRONJ). ${ }^{26,27}$

The objective of this study is to evaluate the effectiveness and safety of ozone therapy conducted with topical applications of ozone as a gel (Ozosan ${ }^{\circledR}$ - Sanipan, Clivio, Italy) in the treatment of osteoradionecrosis of the jaws.

\section{MATERIALS AND METHODS}

Patients included in the study were under radiotherapy for cervical or neck cancer and had, due to radiations, areas of necrotic bone tissue exposed in the jawbone that needed treatment to avoid infection and extension of the necrotic area. The protocol didn't vary depending on the dose of Gray given to the patient or the intake of chemotherapeutic drugs used in combination with radiotherapy.

The study group consisted of 8 patients. Everyone was diagnosed with ORN of the jawbone between 07/2013 and 04/2015 at our Department, for a total of 11 sites of necrotic bone exposure, three patients were diagnosed with more than one site of osteoradionecrosis). All patients involved in the study were treated with ozone-based oil gel using topical applications at the site of bone necrosis. At the time of diagnosis of ORN, the severity was assessed by the classification of He et al.

The exclusion criteria were the impossibility for the patient to show at every scheduled appointment, poor oral hygiene conditions and follow-up of less than one year.

The therapeutic protocol began with professional oral hygiene, followed as soon as possible by the first ozone oil-based gel application. Topical applications included positioning a small amount of gel $(5 \mathrm{~mL})$ over a sterile gauze compressed on the exposed bone area for 10 minutes restricting contact with other oral fluids.

After this period, the gauze is removed and at a distance of 7 days, the procedure is repeated until movement of the necrotic fragment is noted.

Domestic therapy involved topical applications on the necrotic site with sterile gauze of hydrogenated gel preparation 1-2 times a day.

The patients were instructed not to smoke or drink alcoholic beverages during the whole length of the treatment.

Site healing is evaluated with the release of necrotic bone fragment and consequent spontaneous wound closure for a period of 12 months.

The type and location of carcinoma appearance and co-morbidity of patients are shown in Table 1.

\section{RESULTS}

The male/female ratio was $7 / 1$ (Table 1 ). The age of patients at diagnosis of ORN ranged from 54 to 76 years (mean 62 years \pm 7.4 years). For the three patients with a diagnosis of ORN in two different sites, age was considered at the time of diagnosis at the first appearance 


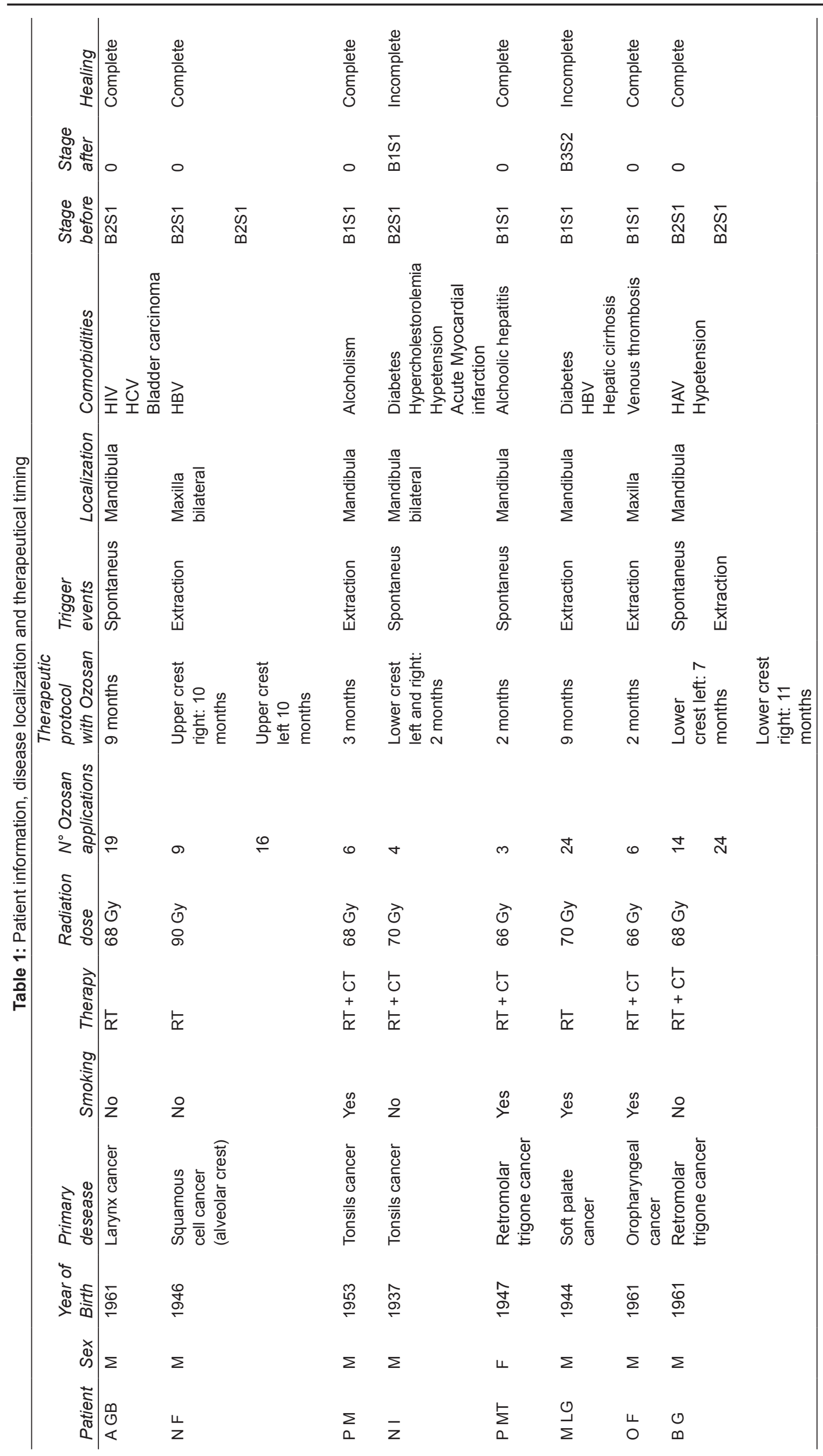


of a necrotic bone area. Five patients had undergone a combination of radiotherapy and chemotherapy, while three patients had undergone radiotherapy alone. The average radiation dose received was $68 \mathrm{~Gy}$ for the radio/ chemotherapy group (min 66 max 70 Gy) and 76 Gy for the radiotherapy group (min 68 max $90 \mathrm{~Gy}$ ). The mean onset of ORN after the end of radiotherapy was 23, 2 months for each site of osteoradionecrosis. 4 out of 8 patients were strong smokers (50\%), and 2 out of 8 patients had problems with alcoholism (25\%).

Five out of eight patients (62.5\%) were diagnosed with a post extractive delayed healing of the surgical wound (Figs 1 and 2). Similarly, a total of seven post-extraction sites on eleven showed an ORN (63.6\%) with dehiscence of the surgical wound and lack of spontaneous closure. In the other cases, ORN occurred spontaneously $(37.5 \%$ of patients and $36.6 \%$ of the total sites with diagnosed ORN). In six sites of ORN the initial stage was B2S1, and in the other five, the initial stage was B1S1. The upper/ lower jaw ratio as the site of the onset of the lesion was 3:8, with a marked predominance of mandibular ORNs sites than those in the upper jaw.

The average number of sessions for Ozosan ${ }^{\circledR}$ ozone gel for each site of osteonecrosis was 12.6. The average number for each patient was 17.3, since three patients showed more than one osteonecrotic spot.

Six patients out of 8 obtained complete healing (75\%), one patient improved his conditions shifting from a stage B2S1 to B1S1 according to He classification. A patient only worsened his conditions shifting from a stage B1S1 to B3S2.

Two patients with three sites affected with no healing received Ozosan ${ }^{\circledR}$ gel, but in the first case, due to the size of the ORN, it was decided to complete surgically the removal of the osteonecrotic bone area with osteoplasty and mucous flap coverage. In the second case, the patient was subjected to hemimandibulectomy.

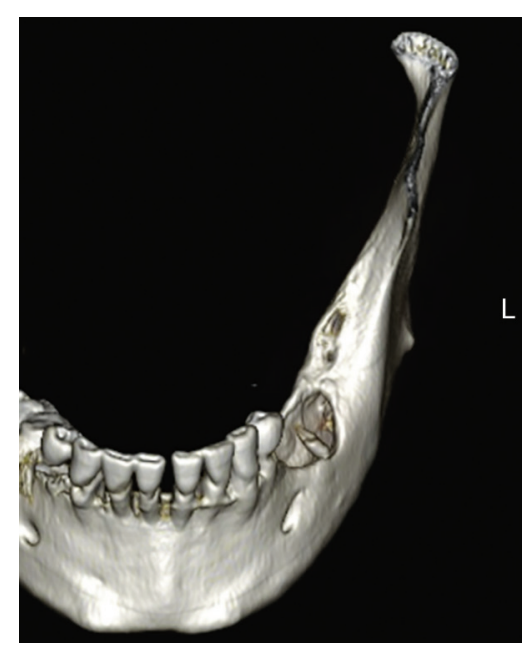

Fig 1: Computerized tomography at T0

\section{DISCUSSION}

Osteoradionecrosis is secondary to permanent damage of the bone-vascular bed and of the osteocytes generated by radiations. It is a bone lesion characterized by a slow progression and impossibility for spontaneous healing. It can't be considered an autonomous disease, but it is the consequence of several local and systemic factors capable of modifying the bony hematic flow and surrounding tissues thus leading to bone exposition.

The trigger event can result from infections in the alveolar processes, tooth extractions or other invasive dental procedures or traumatic events that occurred during the period of radiotherapy or immediately after it. ${ }^{28-30}$ Osteoradionecrosis may also occur spontaneously, and in the present study, the outbreak was spontaneous in four out of the eight patients, even if poor oral hygiene, poor mandibular vascularization, high radiation doses or smoking or alcohol abuse may be predisponent factors.

Many different classifications have been proposed with the aim of standardizing ORN of the jaws (ORNJ) and their treatment. He et al. proposed in 2015 the newest classification, based on the presentation of the disease and radiological findings, in which bone and soft tissue are classified separately, and the results then combined dividing patients with ORNJ in 4 stages (from stage 0 to stage 3 ). The therapeutic strategy is decided on a clinical stage ratio. ${ }^{24}$

Currently, there are various treatment options for ORNJ, but in literature is still unclear which is the best therapy for this undesired complication.

Conservative management is recommended in B0S0 stage according to the classification proposed by He et al. and consists of antibiotic therapy, antiseptics, promotion of dental hygiene, local wound care and hyperbaric oxygen therapy (HBO). In this early stage, bacterial culture and an antibiogram are indicated, though broadspectrum treatment with penicillins, macrolides, and fluoroquinolones has yielded good results. ${ }^{24}$

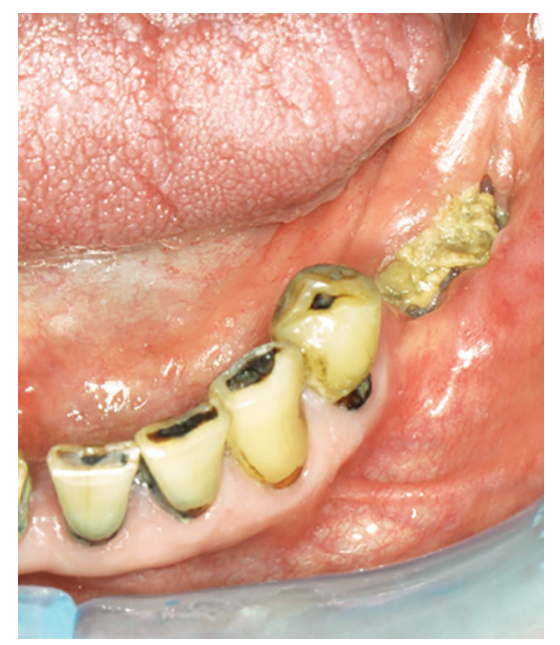

Fig 2: Osteonecrotic lesion at T0 
Surgical treatment in B1 stage is directed to eliminate necrotized bone in order to leave only healthy bone.

At this stage, due to the small size of the defects, after debridement, we preferred a limited and conservative approach, to reduce discomfort and an open surgical procedure that should be critical especially for patients with compromised systemic conditions.

Marginal or segmental resection is required in B2 stage patients. The surgical margins are determined through preoperative CT combined with the appearance of bleeding bone during surgery. For patients who underwent extended segmental resection, reconstruction of the defect is essential to improve both function and aesthetics. In these cases, pretreatment with ozone gel could be useful in reducing side effects or secondary infection of the surgical wound. ${ }^{1,24}$

An alternative treatment strategy is a use of laser therapy, both Low-Level Laser Therapy (LLLT), widely used to stimulate and accelerate cellular response, and Er: YAG/Er, Cr: YSGG. ${ }^{13,31}$

In this study, the Authors selected ozonized oil gel because the application of gel was found to provide a more extended stay in the oral cavity, adequate drug penetration, high efficacy and acceptability for both the patient and the operator, with only minor side effects. ${ }^{32}$

When ozone bounded to the substrate is released promotes an oxidizing action due to a series of reactions of the target organism. ${ }^{33,34}$

Ozone acts on enzymes and nucleic acids in the cytoplasm, and proteins and peptidoglycan in virus capsids. This ozone-induced modification is selective to microbial cells but does not damage human body cells because of their major anti-oxidative ability. ${ }^{35,36}$ RNA of microorganisms, in fact, is degraded into protein subunits by ozonization. Ozone alters the protein capsid first to liberate RNA that may be secondarily inactivated by ozone. Ozone also acts on fungi and protozoa. 37,38

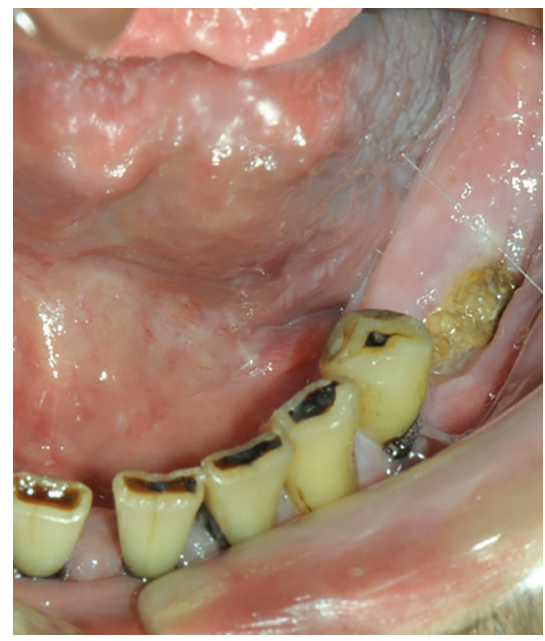

Fig 3: Lesion after 4 applications of ozonated oil gel
Ozone has a positive effect on blood circulation, increasing the concentration of red blood cells and hemoglobin. This localized stimulation of the circulatory system leads to increased oxygenation of tissues, and this effect is more evident in the vessels of smaller diameter (such as vases intraosseous maxillary or capillaries) that fill quickly red blood cells by increasing local microcirculation. The action of stimulation of angiogenesis finally leads to a more represented vascular network. This increase in vascularity of the underlying necrotic bone area causes the formation of granulation tissue (Fig. 3). Along with an adequate curettage and frequent washing with saline solution and hydrogen peroxide, revascularization of the underlying bone causes by the time the spontaneous dislocation of the necrotic fragment, exposing a living tissue that will not be necessary to treat surgically and that will lead to a partial healing of the wound or at the complete closure of the wound (Fig. 4). ${ }^{26,28,39}$

As supported by the literature, many oral cancer patients often have other related pathologies (diabetes, HBV, HCV, HIV) or history of alcohol abuse or are strong smokers, and these factors may have increased chances of developing osteoradionecrosis. Chemotherapy may also intensify the acute and late reactions of the body to radiation damage. ${ }^{40}$ In our patient sample, it was noted that all had co-morbidity related to a possible increase in the risk of developing ORNJ (hepatitis A, B or C, alcoholic hepatitis, HIV, diabetes, venous thrombosis), as well as 4 patients on $8(50 \%)$ were strong smokers, while two out of eight $(25 \%)$ had problems with alcoholism. A marked predominance of mandibular ORNJ was observed in the upper jaw. Literature suggests that the ORNJ affects the lower jaw more frequently than the upper jaw or any other bone in the head circumference. It should be noted that in the present study, cases not solved by ozone therapy with ozonized oil gel were all osteoradionecrosis localized

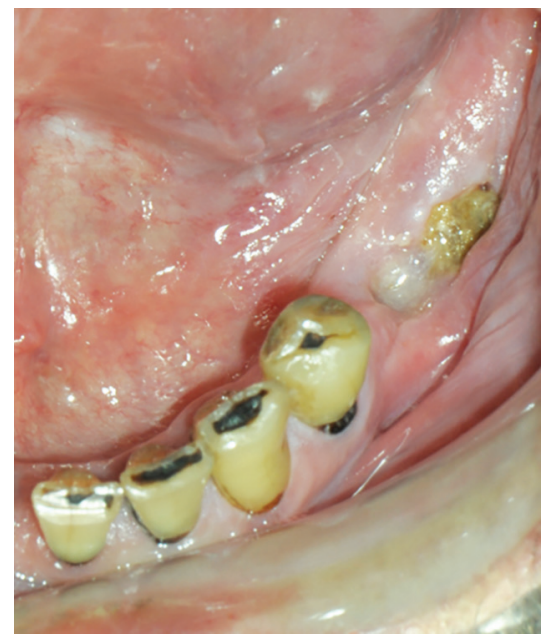

Fig 4: Partial healing after 8 applications of ozonated oil gel 
at mandibular level. These data are also supported by the literature that claims that the ORNJ affects the jaw more frequently than the upper jaw or any other bone in the head circumference, probably because of its relatively reduced vascularization and/or its very compact bone structure. ${ }^{19}$ This predisposition is also due to the obliteration of the lower alveolar artery and the concomitant difficulty of revascularization by facial artery branches because of radiation-induced fibrovascular necrosis. ${ }^{17}$ The male population included in the present study was of 8 patients, compared to only one female patient. This data is also in agreement with the literature: Reuther et al. In a retrospective study on 830 patients show an incidence three times greater for men than for women. This may be due to a higher percentage of alcohol and tobacco abuse in male ORN patients than in female ORN patients. ${ }^{19}$

\section{CONCLUSION}

There is a wide variation in recommendations and guidelines in international literature for the treatment of ORNJ. A universally accepted approach to the treatment of ORNJ should be developed.

Authors suggest a therapeutic protocol based upon minimally invasive debridement of the wound, antibiotic and antimycotic therapy with ozonated oil gel adopting ozone itself as regenerating factor for tissues. In $87.5 \%$ of cases, the results have confirmed the effectiveness of this kind of approach, with six patients healed completely and one with improvement in the clinical staging (Fig. 5).

According to the Authors' experience the benefits of using ozone as stimulation of local revascularization through the enhancement of angiogenesis and fibroblasts, make the use of ozonated oil gel an ideal aid in the noninvasive treatment of osteoradionecrosis of the jaw if used in conjunction with continuous cleansing of the wound by the patient.

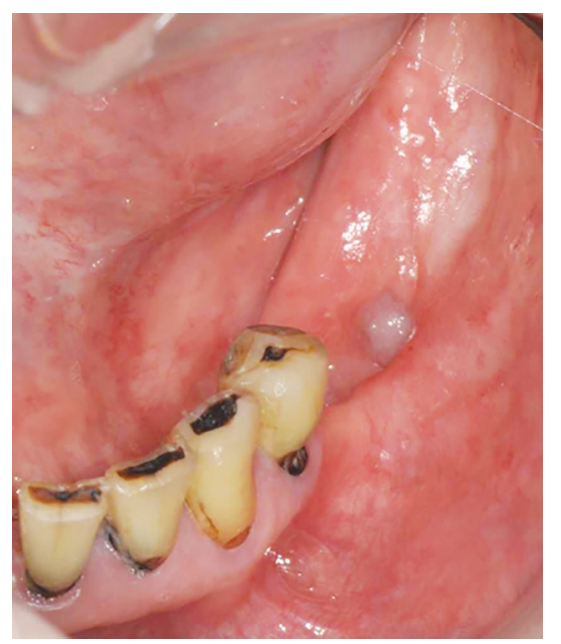

Fig 5: Complete wound healing with reepithelialisation of oral mucosa after 19 applications of Ozosan ${ }^{\circledR}$.
The oil-based gel ozone formulation concerning its administration as a gas or as ozonized water showed the ability to remain in stable contact with the bone surface for a more extended period.

The use of ozone gel should be incorporated in the treatment of less complex osteoradionecrosis that does not involve invasive surgical approach, sometimes responsible for a worsening of the clinical condition of the patient. The limitation of this protocol is that, compared to an exclusively surgical approach, a longer time is required for the necrotic portion of the bone to be removed.

\section{CLINICAL SIGNIFICANCE}

The use of a noninvasive, local, easy to use and selfadministered medication able to control, reduce and eventually solve this severe complication in an oncologic patient should be useful and included in routine clinical protocols.

\section{REFERENCES}

1. Silvestre-Rangil J, Silvestre FJ. Clinico-therapeutic management of osteoradionecrosis: A literature review and update. Med Oral Patol Oral Cir Bucal. 2011;16(7):e900-904.

2. Nabil S, Samman N (2011) Incidence and prevention of osteoradionecrosis after dental extraction in irradiated patients: a systematic review. Int J Oral Maxillofac Surg 40(3): 229-243.

3. Marx RE, Johnson RP, Kline SN (1985) Prevention of osteoradionecrosis: a randomized prospective clinical trial of hyperbaric oxygen versus penicillin J Am Dent Assoc 111(1):49-54.

4. Porcaro G, Busa A, Bianco E, Caccianiga G, Maddalone M. Use of a Partial-thickness Flap for Guided Bone Regeneration in the Upper Jaw. J Contemp Dent Pract. 2017 Dec 1;18(12):1117-1121.

5. Ivanović, M., Jovičić, O., Mandić, J., Bogetić, D., Maddalone, M. Prevention of oral diseases in children with acute leukaemia 2011 Srpski Arhiv za Celokupno Lekarstvo139(3-4), pp. 242-247

6. Ivanović, M., Jovičić, O., Mandić, J., Bogetić, D., Maddalone, M. Oral manifestations of acute leukaemia Srpski Arhiv za Celokupno 2011 139(1-2), pp. 103-106

7. Maddalone M, Ferrari M ,Stanizzi A, Barrila' S, Arrigoni S. Use of miniscrew implants in orthodontic distal movement [Utilizzo delle miniviti nelle meccaniche ortodontiche di distalizzazione] Dental Cadmos 2010; 8:97-105

8. Maddalone M, Ferrari M, Barrila' S, Arrigoni S ,Stanizzi A. Intrusive mechanics in orthodontics with the use of TAD's [Utilizzo delle miniviti nelle meccaniche ortodontiche di intrusione ] Dental Cadmos 2010; 7:97-106

9. Maddalone M, The association between the psychological status and the severity of facial deformity in orthognathic patients Angle Orthodontist 2012; 82(3) :396-402

10. Citterio F, Pellegatta A, Citterio CL, Maddalone M. Analysis of the apical constriction using micro-computed tomography and anatomical sections. In: Giornale Italiano Di Endodonzia $2014 ; 28: 41-45$.

11. Venino PM, Citterio CL, Pellegatta A, Ciccarelli M, Maddalone M. A Micro-computed Tomography Evaluation of the 
Shaping Ability of Two Nickel-titanium Instruments, HyFlex EDM and ProTaper Next. J Endod. 2017;43(4):628-632.

12. Maddalone M, Gagliani M, Citterio CL, Karanxha L, Pellegatta A, Del Fabbro M. Prevalence of vertical root fractures in teeth planned for apical surgery. A retrospective cohort study Int Endod J. 2018 Feb 25. doi: 10.1111/iej.12910. [Epub ahead of print]

13. Chrcanovic BR, Reher P, Sousa AA, Harris M (2010) Osteoradionecrosis of the jaws-a current overview--part 1: Physiopathology and risk and predisposing factors. Oral Maxillofac Surg 14(1):3-16.

14. Oh HK, Chambers MS, Martin JW, Lim HJ, Park HJ (2009) Osteoradionecrosis of the mandible: treatment outcomes and factors inflencing the progress of osteoradionecrosis. J Oral Maxillofac Surg 67(7):1378-1386.

15. Manimaran K, Sankaranarayanan S, Ravi VR, Elangovan S, Chandramohan M, Mahendra Perumal S (2014) Treatment of osteoradionecrosis of mandible with bone marrow concentrate and with dental pulp stem cells. Ann Maxillofac Surg 4(2):189-192.

16. Batinjan G, Filipovic Zore I, Vuletic M, Rupic I (2014) The use of ozone in the prevention of osteoradionecrosis of the jaw. Saudi Med J 35(10):1260-1263.

17. Regaud C (1922) Sur la sensibilite du tissu osseux normal vis-a-vis des rayons $\mathrm{X}$ et gamma et sur la mecanisme de l'osteoradionecrose. R Soc Seances Soc Biol Fil 87: 629-932.

18. Thiel HJ (1989) Osteoradionecrosis. Etiology, pathogenesis, clinical aspects and risk factors. Radiobiol Radiother 30(5):397-413.

19. Reuther T, Schuster T, Mende U, Kubler A (2003) Osteoradionecrosis of the jaws as a side effect of radiotherapy of head and neck tumour patients-a report of a 30 year retrospective review. Int J Oral Maxillofac Surg 32(3):289-295

20. Manzoni L, Rossi E, Fratto G (2015) Management of osteonecrosis of the jaws induced by radiotherapy in oncological patients: preliminary results. European Review for Medical and Pharmacological Sciences 19(2):194-200.

21. Nessi R, Viganò L (2004) I raggi X e la materia vivente. In: Nessi R, Viganò L (eds). Radiologia Odontostomatologica. Piccin Nuova Libraria, Padova, 51-54.

22. Sulaiman F, Huryn JM, Zlotolow IM (2003) Dental extractions in the irradiated head and neck patient: a retrospective analysis of Memorial Sloan-Kettering Cancer Center protocols, criteria, and end results. J Oral Maxillofac Surg 61(10):1123-1131.

23. Tong AC, Leung AC, Cheng JC, Sham J (1999) Incidence of complicated healing and osteoradionecrosis following tooth extraction in patients receiving radiotherapy for treatment of nasopharyngealcarcinoma. Aust Dent J 44(3):187-194.

24. He Y, et al. (2015) Retrospective analysis of osteoradionecrosis of the mandible: proposing a novel clinical classification and staging system, Int J Oral Maxillofac Surg 44(12):15471557.
25. Saini R (2011) Ozone therapy in dentistry: A strategic review. J Nat Sci Biol Med Jul;2(2):151-153.

26. Agrillo A, Petrucci MT, Tedaldi M, Mustazza MC, Marino SM, Gallucci C, Iannetti G (2006) New Therapeutic Protocol in the Treatment of Avascular Necrosis of the Jaws. J Craniofac Surg 17(6):1080-1083.

27. Agrillo A, Sassano P, Rinna C, Priore P, Iannetti G (2007) Ozone Therapy in Extractive Surgery on Patients Treated with Bisphosphonates. J Craniofac Surg 18(5):1068-1070.

28. Agrillo A, Filiaci F, Ramieri V, Riccardi E, Quarato D, Rinna C et al. (2012) Bisphosphonate-related osteonecrosis of the jaw (BRONJ): 5 year experience in the treatment of 131 cases with ozone therapy European Review for Medical and Pharmacological Sciences 16(12):1741-1747.

29. Ficarra G (2006). Alterazioni orali causate da agenti fisici e chimici e da farmaci. In: Ficarra G (ed). Manuale di patologia e medicina orale. Milano: The McGraw-Hill Companies, Milano, 459-470.

30. Rathy R, Sunil S, Nivia M (2013) Osteoradionecrosis of mandible: Case report with review of literature. Contemp Clin Dent Apr-Jun;4(2):251-253.

31. Caccianiga G, Paiusco A, Perillo L, Nucera R, Pinsino A, Maddalone M (2017) Does Low-Level Laser Therapy enhance the efficiency of orthodontic dental alignment? Results from a randomized pilot study. Photomed and Laser Surg 35(8):421-426.

32. Dayas A, Boughton BJ, Das BC. Ozone killing action against bacterial and fungal species; mcrobiological testing of a domestic ozone generator. J Clin Pathol 1983;36(10):1102-1104.

33. Muller P, Guggenheim B, Shmidlin P (2007) Efficacy of gasiform ozone and photodynamic therapy on a multispecies oral biofilm in vitro. Eur J Oral Sci 115(1):77-80.

34. Kumar T, Arora N, Puri G, Aravinda K, Dixit A, Jatti D (2016) Efficacy of ozonized olive oil in the management of oral lesions and conditions: A clinical trial. Contemp Clin Dent 7:51-54.

35. Scott DBM, Lesher EC (1963) Effect of ozone on survival and permeability of escherichia coli. J Bacteriol 85:567-576.

36. De Almeida NR, Beatriz A, Micheletti AC, De Arruda EJ (2012)Ozonized vegetable oils and therapeutic properties: A review. Orbital Elec J Chem 4(4):313-326.

37. Geweely N (2006) Antifungal activity of ozonized olive oil (Oleozone). Int J Agri Biol 8(5):670-675.

38. Seidler V, Linetskiy I, Hubálková $H$, Stanková H, Smucler R, Mazánek J (2008)Ozone and its usage in general medicine and dentistry. A review article. Prague Med Rep 109(1):5-13.

39. Brozoski MA, Lemos CA, Da Graca Naclerio-Homem M, Deboni MCZ (2014) Adjuvant acqueous ozone in the treatment of biphosphonate induced necrosis of the jaws: report of two cases and long term follow up. Minerva Stomatol 63(1-2):35-41.

40. Studer G, Grätz KW, Glanzmann C (2004) Osteoradionecrosis of the mandibula in patients treated with different fractionations. Strahlenther Onkol 180(4):233-240. 